\title{
Effect of a Handover Tool on Efficiency of Care and Mortality for Interhospital Transfers
}

\author{
Cecelia N. Theobald, MD, MPH${ }^{1,2}$, Neesha N. Choma, MD, MPH ${ }^{1}$, Jesse M. Ehrenfeld, MD, MPH${ }^{3}$, Stephan Russ, MD, MPH4, \\ Sunil Kripalani, MD, MSc ${ }^{1,2}$
}

${ }^{1}$ Department of Medicine, Vanderbilt University Medical Center, Nashville, Tennessee; ${ }^{2}$ Center for Clinical Quality and Implementation Research, Vanderbilt University Medical Center, Nashville, Tennessee; ${ }^{3}$ Departments of Anesthesiology, Surgery, Biomedical Informatics, and Health Policy, Vanderbilt University Medical Center, Nashville, Tennessee; ${ }^{4}$ Department of Emergency Medicine, Vanderbilt University Medical Center, Nashville, Tennessee

\begin{abstract}
BACKGROUND: Interhospital transfer is frequent, and transferred patients experience delays in the provision of care and higher mortality rates when compared to patients directly admitted. The interhospital handover is a key opportunity to improve care but has not been evaluated.
\end{abstract}

OBJECTIVE: To determine the effect of a universal handover tool on timeliness of care, length of stay (LOS), and mortality among interhospital transfer patients.

DESIGN, SETTING, AND PATIENTS: Retrospective cohort of patients transferred to an academic medical center between July 1, 2009 and December 31, 2010 with interrupted time-series design.

INTERVENTION: One-page handover tool containing information critical for immediate patient care instituted hospital-wide on July 1, 2010. The handover tool was completed by the transferring physician and available for review before patient arrival.
MEASUREMENTS: Time-to-admission order entry, LOS after transfer, in-hospital mortality

RESULTS: There was no significant change in the time-to-admission order entry after implementation (47 minutes vs. 45 minutes, adjusted $P=0.94)$. There was a nonstatistically significant reduction in LOS after implementation (6.5 days vs. 5.8 days, adjusted $P=0.06$ ). In-hospital mortality for transfer patients declined significantly in the postintervention period from $12.0 \%$ to $8.9 \%$ (adjusted odds ratio, 0.68; 95\% confidence interval, $0.47-0.99, P=0.04$ ). There was no change in mortality for the concurrent control group.

CONCLUSION: Implementation of a standardized handover tool for interhospital transfer was feasible and may be associated with significant reductions in length of stay and mortality. Widespread adoption of similar tools may improve outcomes in this high-risk population. Journal of Hospital Medicine 2017;12:23-28. (c) 2017 Society of Hospital Medicine
The transfer of inpatients between hospitals for specialized services is common, affecting nearly $10 \%$ of all Medicare admissions ${ }^{1}$ and $4.5 \%$ of all critical care hospitalizations. ${ }^{2} \mathrm{At}$ tertiary referral centers, $49 \%$ of medical intensive care unit (ICU) admissions are transferred from another hospital. ${ }^{3}$

Transfer patients have longer length of stay (LOS) than patients admitted directly from the emergency department or clinic. Among patients initially admitted to an ICU, transfer patients spend 1 day to 2.2 more days in the ICU and an additional 2 days to 4 more days total at the receiving hospital. ${ }^{4,5}$ Furthermore, transfer patients have higher mortality than nontransferred patients by $4 \%$ to $8 \% .^{3-5}$ Even after adjustment for case mix and comorbid disease, interhospital transfer is an independent predictor of both ICU death and LOS. ${ }^{6,7}$ As a result, interhospital transfer has been associated with a $\$ 9600$ increase (on average) in hospital costs. ${ }^{4}$

Despite evidence detailing patient handovers as a key time when poor communication can lead to delays in care and sig-

\footnotetext{
*Address for correspondence and reprint requests: Cecelia N. Theobald, Division of General Internal Medicine and Public Health, 1214 21st Ave. S, Medical Center East-NT, 7th floor Suite IV, Nashville, TN 37232; Telephone: 615-9363216; Fax: 615-936-3156; E-mail: cecelia.theobald@vanderbilt.edu
}

Additional Supporting Information may be found in the online version of this article. Received: May 4, 2016; Revised: July 28, 2016; Accepted: August 4, 2016 2017 Society of Hospital Medicine DOI 10.1002/jhm.2669 nificant patient risk, ${ }^{8-10}$ most studies have focused on hospital discharge or change of shift, and scant effort has been dedicated to improving the interhospital handover. The process of interhospital transfer is often prolonged and discontinuous, ${ }^{11}$ commonly including delays of more than 24 hours between initiation and completion. This frequently precludes direct physician-to-physician contact at the time of transfer, and physicians rely on the discharge/transfer summary. ${ }^{12}$ Yet discharge summaries are frequently absent or incomplete, ${ }^{13}$ and often lack information for high-risk treatments such as systemic anticoagulation. ${ }^{14}$ The traditional reliance on discharge summaries for handover communication requires interpretation of unstandardized documentation and increases the risk for miscommunication, delays, and error.

To improve communication, we developed a 1-page handover tool for all inbound adult interhospital transfers to our academic medical center. We sought to determine whether implementation of this standardized handover tool improved the timeliness of initial care, LOS, and mortality among interhospital transfer patients.

\section{METHODS}

\section{Study Design, Setting, Population}

We conducted a retrospective cohort study of patients transferred into Vanderbilt University Hospital (VUH), an adult 626-bed quaternary care academic medical center in Nash- 
ville, Tennessee. The Vanderbilt University Institutional Review Board approved this study.

\section{Population}

We selected for inclusion all patients age 18 or older who were transferred into VUH between July 1, 2009 and December 31, 2010. We excluded patients whose transfer was routed outside the main VUH Patient Flow Center as well as patients who did not arrive alive at VUH. We also excluded patients transferred to the emergency department and patients admitted to obstetrics, burn, or trauma services, because these admitting services did not initially use the handover tool. Patients were followed for the duration of their hospitalization at VUH.

\section{Intervention}

The 1-page handover tool was developed with multidisciplinary physician input from house staff; medical directors from intensive care, neurology, and surgery; and the chief of staff. The tool was structured on the SBAR model (Situation, Background, Assessment, and Recommendation). ${ }^{15}$ Fields on the handover tool were limited to those deemed critical for immediate patient care and designed for 1 tool to be used for both ICU and non-ICU transfers. Fields included primary diagnosis; allergies; use and last dose of anticoagulants, vasopressors, sedative/paralytics, and antibiotics; isolation needs; indwelling devices; recent operations/procedures; code status; emergency contact information; problem list; active medication list; vital signs; pertinent exam; imaging; lab findings; and overall reason for transfer.

The handover tool was completed by the physician at the transferring hospital, faxed to $\mathrm{VUH}$, and immediately scanned into the electronic record, allowing the receiving physicians to review information before patient arrival. Use of the tool was piloted first with 2 referring hospitals in April 2010 and universally recommended but not compulsory for all adult patients routed through the main VUH Patient Flow Center beginning July 1, 2010. Immediately before full implementation, the chief of staff sent letters to leadership of the 40 highest volume referral hospitals, highlighting the institutional goal of improving hand-off communication, framing completion of the tool as a step in the transfer acceptance process, and providing contact information for questions, feedback, or concerns. To ensure the tool was a standard part of the transfer process, the VUH Patient Flow Center maintained the responsibility of faxing the form to the outside facility and monitoring its receipt. The tool was processed in the same manner as other faxed patient records and treated as a part of the formal medical record to meet all standards for the Health Insurance Portability and Accountability Act (HIPAA) and medicolegal compliance. The medical center also has a separate cardiac transfer center where the handover tool was not implemented owing to its specialized workflow.

\section{Data Source}

The VUH Patient Flow Center maintains a database of all patients for whom transfer to VUH is requested, including information on the requesting hospital and the duration of transfer process. Outcome data and patient characteristics were extracted from the Enterprise Data Warehouse. Data related to comorbid illness were extracted from the Perioperative Data Warehouse, an IRB-approved data registry.

\section{Measures}

We evaluated 3 outcomes. First, we defined 2 measures of the timeliness of initial care, the time from arrival at VUH until entry of an admission order, and the time from arrival until entry of the first antibiotic order. Only antibiotics ordered within the first 36 hours of admission were included. Second, we evaluated the total LOS after transfer to VUH and the ICU LOS for patients transferred into an ICU setting. Finally, we examined in-hospital mortality at VUH. These metrics were chosen for their broad applicability across patient groups and feasibility of data capture. Length of stay and mortality also represent final common pathways for avoidance of complications. Specific patient safety indicators and complications were not abstracted due to their low frequency and burden of data collection. Due to system changes in our cost accounting systems, we were not able to obtain cost data pre- and postimplementation that provided meaningful comparisons.

Patient covariates included age, gender, payer, and Elixhauser comorbidity index as modified by van Walraven, ${ }^{16}$ calculated based on the admission of interest and the previous 365 days. We also examined admission characteristics including location (ICU vs. non-ICU), admitting service (medicine, surgery, neurology, or gynecology), and shift of arrival (day, 7:00 AM to 6:00 PM; or night, 6:00 PM to 7:00 $\mathrm{PM})$. Finally, we examined duration of the transfer process (ie, time between transfer request and arrival at $\mathrm{VUH}$ ) and the volume of the transferring hospital (high was defined as 3 or more transfers to $\mathrm{VUH}$ per year).

\section{Statistical analysis}

Patient characteristics before and after implementation of the handover tool were compared using Pearson's chi-square test and Fisher exact test for categorical variables and using Student $\mathrm{t}$ test and the Wilcoxon rank sum test for continuous variables. The outcome variables of time to admission order entry, time to antibiotic order entry, LOS, ICU LOS, and in-hospital mortality were compared between the before- and after-intervention time periods, using the Wilcoxon rank sum test for continuous outcomes and Pearson's chi-square test for in-hospital mortality.

To account for temporal trends, the effect of the handover tool on time-to-admission order entry, hospital LOS, and mortality was measured using an interrupted time-series design with segmented linear regression analysis. ${ }^{17}$ The study period was divided into 2 -week intervals, with 26 time periods in the pre-intervention period and 13 time periods in the postintervention period. Expected rates for the postintervention time periods were projected from the pre-intervention data using a linear regression model. To assess the observed effect 
of the intervention, rates from the postintervention periods were compared with these projected rates, assuming continuation of the trend. Restricted cubic spline models were also fit for time-to-admission order and hospital LOS; however, the F-statistics for these models were not significant, suggesting the linear regression provided a more appropriate model.

To further account for potential confounding of outcomes by comorbid disease and other patient factors, multivariate linear regression models assessed change in timeliness and LOS with implementation of the intervention. A multivariate logistic regression model was used to assess change in mortality with intervention implementation. All models adjusted for age, gender, payer, comorbid illness, admitting team, shift of arrival (day vs. night), transfer duration, volume of transferring hospital, and ICU status. Outcomes were further adjusted for calendar month to account for temporal trends in house staff efficiency. Because the cardiac transfer center did not adopt the use of the transfer tool, we evaluated adjusted in-hospital mortality for these patients as a concurrent control group.

All statistical testing was 2 -sided at a significance level of 0.05. All analyses were conducted using STATA 12.1 statistical software (StataCorp LP, College Station, Texas).

\section{RESULTS}

Of 10,325 patients for whom transfer to VUH was requested during the study period, 1715 met inclusion criteria, including 798 patients (46.5\%) initially admitted to an ICU setting. Specific patient exclusions are detailed in the Supplemental Figure; the majority of exclusions were due to patients being transferred directly to the emergency department setting. Table 1 summarizes patient characteristics before and after implementation of the handover tool. The median age was 57 years, with $48.6 \%$ male patients. Accepting services included medicine (56\%), surgery (34\%), neurology $(9 \%)$, and gynecology (1\%). The median duration of transfer was 8 hours, and the majority (93\%) of patients came from higher volume transferring hospitals. Most (65\%) of patients were admitted during night shift. The median modified Elixhauser comorbidity index was 11 (range of possible scores, -19 to 89 ). A slightly higher proportion of patients admitted postimplementation of the handover tool came from higher volume transferring hospitals; otherwise, there were no significant differences between the pre- and postintervention groups.

Vanderbilt University Hospital received transfers from more than 350 unique facilities in more than 25 U.S. states during the overall study period. During the postintervention period, adherence to the handover process was excellent, with more than $85 \%$ of patients having a completed handover tool available in their medical record at the time of transfer. The remaining $15 \%$ had either incomplete forms or no form.

\section{TABLE 1. Patient Characteristics Before and After Implementation of the Handover Sheet}

\begin{tabular}{|c|c|c|c|}
\hline Characteristic & $\begin{array}{l}\text { Pre-intervention } \\
\quad(n=1105)\end{array}$ & $\begin{array}{l}\text { Postintervention } \\
\quad(n=610)\end{array}$ & $P$ value \\
\hline Age, median (IQR) & $56.9(45-67)$ & $57.4(44-68)$ & 0.85 \\
\hline Male & $47.5 \%$ & $50.7 \%$ & 0.21 \\
\hline Payer & & & 0.66 \\
\hline Commercial & $37.7 \%$ & $35.6 \%$ & \\
\hline Medicaid & $11.2 \%$ & $10.5 \%$ & \\
\hline Medicare & $45.5 \%$ & $48.7 \%$ & \\
\hline Self-pay & $5.6 \%$ & $5.3 \%$ & \\
\hline ICU admission (\%) & $46.4 \%$ & $46.7 \%$ & 0.91 \\
\hline Service & & & 0.67 \\
\hline Medicine & $55.4 \%$ & $56.3 \%$ & \\
\hline Surgery & $33.9 \%$ & $34.0 \%$ & \\
\hline Neurology & $8.9 \%$ & $8.7 \%$ & \\
\hline Gynecology & $1.7 \%$ & $1.0 \%$ & \\
\hline Duration of transfer (hr), median (IQR) & $8.1(4.9,19.3)$ & $8.2(5.2,16.9)$ & 0.69 \\
\hline \multicolumn{4}{|l|}{ Volume of transferring hospital } \\
\hline$\geq 3$ transfers per year & $92.4 \%$ & $95.2 \%$ & 0.027 \\
\hline$<3$ transfers per year & $7.6 \%$ & $4.8 \%$ & \\
\hline \multicolumn{4}{|l|}{ Time of arrival } \\
\hline Day shift (7:00 AM - 6:00 PM) & $35.7 \%$ & $34.3 \%$ & 0.56 \\
\hline Night shift (6: 00 PM - 7:00 PM) & $64.3 \%$ & $65.7 \%$ & \\
\hline Modified Elixhauser index, median (IQR)a & $11(4-19)$ & $11(2-19)$ & 0.85 \\
\hline
\end{tabular}

aRange of possible scores: -19 to 89.

NOTE: Abbreviations: ICU, intensive care unit; IQR, interquartile range; LOS, length of stay. 
TABLE 2. Effect of Handover Sheet Implementation on Timeliness of Care, LOS, and Mortality

\begin{tabular}{lcccc}
\hline Outcome & $\begin{array}{c}\text { Pre-intervention } \\
(\mathrm{n}=1105)\end{array}$ & $\begin{array}{c}\text { Postintervention } \\
(\mathrm{n}=610)\end{array}$ & $\begin{array}{c}\text { Unadjusted } \\
P \text { value }\end{array}$ & $\begin{array}{c}\text { Adjusted } \\
P \text { value }\end{array}$ \\
\hline Time to admission order entry (min) & $47(20,92)$ & $45(18,87)$ & 0.36 & 0.94 \\
\hline Time to antibiotic ordera $(\min ; \mathrm{n}=1117)$ & $199(78,524)$ & $202(90,492)$ & 0.18 & 0.91 \\
\hline Hospital LOS (d) & $6.47(3.4,11.7)$ & $5.81(3.1,10.9)$ & 0.38 & 0.06 \\
\hline ICU LOS (d; $\mathrm{n}=793)$ & $4.34(2.2,9.0)$ & $4.55(2.7,8.8)$ & 0.04 & 0.99 \\
\hline Inhospital mortality & $12.0 \%$ & $8.9 \%$ & 0.04 \\
\hline
\end{tabular}

a Limited to those patients who received antibiotic order within first 36 hours of admission. Values for LOS and time are presented as median (IQR). Multivariate model includes adjustment for age, gender, payer, admitting team, ICU status, time of admission, modified Elixhauser index, duration of transfer, and volume of transferring hospital; timeliness outcomes were further adjusted for calendar month.

NOTE: Abbreviations: ICU, intensive care unit. IQR, interquartile range; LOS, length of stay.

\section{Timeliness of Initial Care}

There was no change in either the median time-to-admission order entry after implementation ( 47 vs. 45 minutes, unadjusted $P=0.36)$ or time to antibiotic order entry $(199$ vs. 202 minutes; unadjusted $P=0.81$; Table 2).

In the time-series analysis, the pre-intervention period did not have a significant temporal trend in median time-to-admission order entry ( $\beta$-coefficient $=-0.27 ; 95 \%$ confidence interval $[\mathrm{CI}]-0.85$ to $0.31 ; \mathrm{R} 2=0.04 ; P=0.34$; Figure $1 \mathrm{~A}$ ). The postintervention period showed a trend toward a reduction in median time-to-admission order entry ( $B$-coefficient $=-1.39 ; 95 \% \mathrm{CI}-2.92$ to $0.15 ; \mathrm{R} 2=0.27 ; P=0.07)$. There was no significant difference between the actual time-to-admission order entry in the postintervention period when compared to the projected rates from the pre-intervention period $(P=0.18)$.

After multivariate adjustment, the postintervention time period was not associated with any significant change in the median time-to-admission order entry $\left(P=0.94, R^{2}=0.09\right)$ nor time-to-antibiotic order entry $\left(P=0.91 ; \mathrm{R}^{2}=0.08\right.$; Table 2).

\section{Length of Stay}

Hospital LOS demonstrated a nonstatistically significant decline after implementation of the handover tool from 6.47 days to 5.81 days (unadjusted $P=0.18$; Table 2). There was no significant change in ICU LOS postintervention (4.34 days to 4.55 days; $P=0.38$ ).

In time series analysis, hospital LOS did not have a significant temporal trend in either the pre-intervention pe$\operatorname{riod}(B$-coefficient $=0.00094 ; 95 \% \mathrm{CI},-0.07$ to $0.07 ; \mathrm{R} 2=$ $0.00 ; P=0.98$ ) or the postintervention period ( $B$-coefficient $=0.09 ; 95 \% \mathrm{CI},-0.07$ to $0.25 ; \mathrm{R} 2=0.13 ; P=0.23$; Figure 1B). Similarly, there was no significant difference between the actual and projected hospital LOS after implementation of the handover tool $(P=0.31)$.

After multivariate adjustment, the postintervention time period was associated with a trend toward reduction in overall LOS $\left(P=0.06 ; \mathrm{R}^{2}=0.07\right)$ but no significant change in $\operatorname{ICU} \operatorname{LOS}\left(P=0.99 ; \mathrm{R}^{2}=0.09\right)$.

\section{Mortality}

In-hospital mortality declined significantly from $12.0 \%$ in the pre-intervention period to $8.9 \%$ in the postintervention period $(P=0.04$; Table 2$)$. In time-series analysis, mortality did not have a significant trend in the pre-intervention period (B-coefficient $=0.00017,95 \% \mathrm{CI},-0.0020$ to 0.0024 ; $P=0.878)$ and had a trend toward reduction in the postintervention period $(B$-coefficient $=-0.0032 ; 95 \% \mathrm{CI},-0.0091$ to $0.0027 ; P=0.255$; Figure $1 C$ ) but did not reach statistical significance due to relatively small numbers of deaths in each individual time period.

After multivariate adjustment, the postintervention period was associated with overall lower odds of mortality among transfer patients when compared with the pre-intervention period (adjusted OR 0.68; 95\% CI, $0.47-0.99 ; \mathrm{R}^{2}=0.21$; $P=0.04$; Figure 2). Among the concurrent control group of patients routed through the cardiac transfer center, there was no significant change in mortality between the pre- and postintervention periods (adjusted OR 1.31; 95\% CI, 0.88 $\left.1.93 ; \mathrm{R}^{2}=0.28 ; P=0.18\right)$.

\section{DISCUSSION}

We developed a simple 1-page handover tool for interhospital transfer patients and aimed to improve timeliness, efficiency, and outcomes of care at the receiving hospital. Implementation of the handover tool was feasible and well accepted by transferring physicians despite a geographically large and diverse transfer network. Although implementation did not substantially improve measures of the timeliness of initial care among transfer patients, we noted a nonsignificant trend toward reduced LOS postintervention.

We observed a substantial and statistically significant reduction in mortality among transfer patients after implementation of the handover tool that persisted after controlling for time trends, comorbid illness, and several other patient factors. This effect was not seen in a concurrent control group of cardiac transfer patients for whom the handover tool was not implemented. Standardizing communication regarding high-risk clinical care processes may be responsible for the observed mortality reduction, similar to improve- 
ments seen in other small pilot studies. ${ }^{18}$ We acknowledge that the magnitude of the improvement in mortality is more than might be expected from the handover tool alone and could be due to chance.

In this initial evaluation, it was not feasible to determine whether information provided in the handover tool helped

A

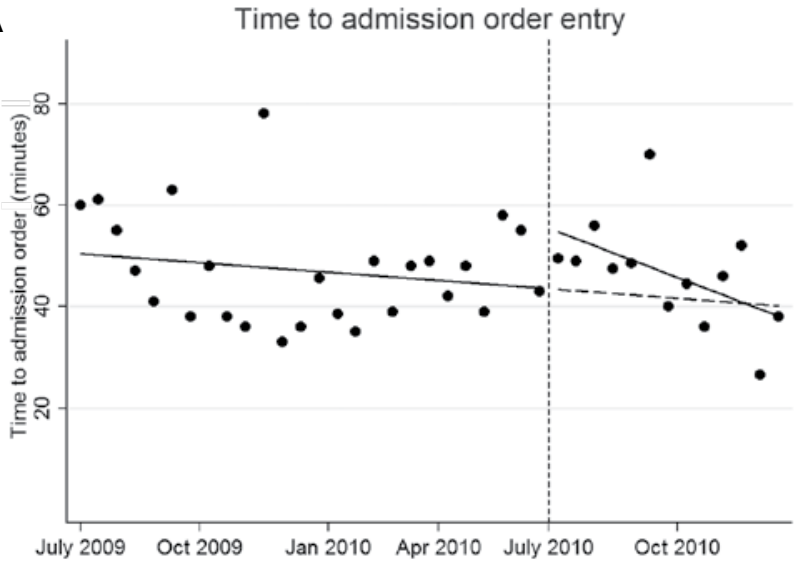

B

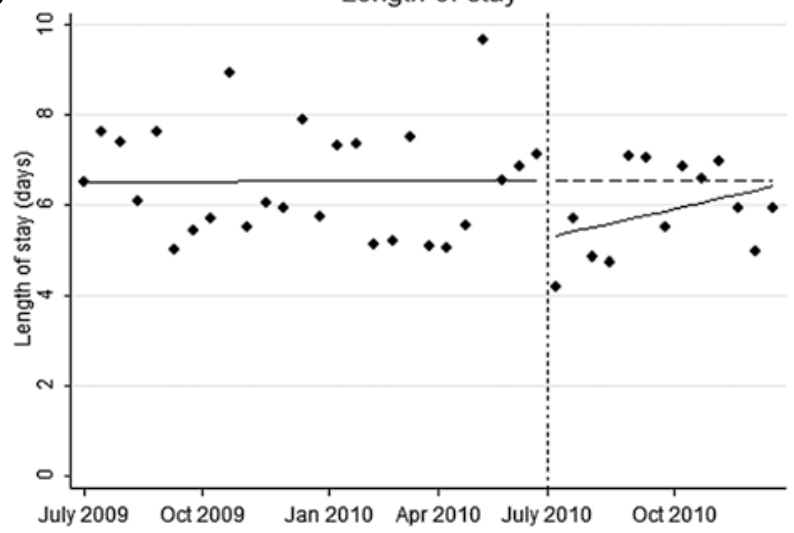

C

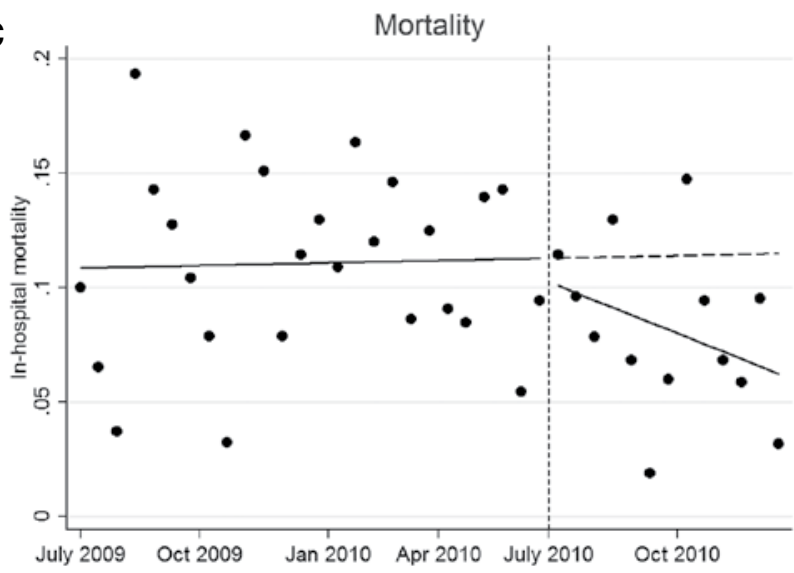

FIG. 1A-1C. Interrupted time series analysis of timeliness of order entry (A), length of stay (B), and mortality (C).

NOTE: Segmented regression analysis of median values during 2-week intervals from July 2009 to December 2010. The baseline period trend was projected into the intervention period to display expected values without implementation of the intervention. The vertical dashed line demarcates the pre- and postintervention periods. avert specific complications that could affect mortality, such as complications related to the use of ventilators, high-risk medications, or indwelling devices. Assessment of additional patient safety indices such as code events, unplanned ICU transfers, and medication errors could also help clarify the effect of the handover tool on patient-safety outcomes, and future work should include these metrics as well. Alternately, the improvement in mortality may result from other unmeasured processes that occurred concurrently and verification of this finding should be completed in other settings.

\section{CONCLUSION}

More work is needed to determine suitable process and outcome measures for interhospital transfers. Most literature has focused on cost and LOS at the exclusion of more proximal measures of initial care. ${ }^{3-7}$ The Institute of Medicine has identified timeliness as 1 of the 6 aims for care delivery redesign, ${ }^{19}$ yet standardized timeliness outcomes do not exist across broad inpatient populations. We chose to monitor the time-to-admission order entry and time-to-antibiotic order entry as 2 indicators of timeliness that would be applicable to a variety of patients. The lack of change in these selected measures should prompt examination for other measures of efficiency, including those that affect nontransferred patients. It is possible that nontransferred patients cared for by the same physician also benefit from fewer delays or disruptions and experience increased efficiency of care if transfer patient communication is improved. Further work is necessary to understand whether other measures of timely initial patient care may be more suitable.

The use of a time-series design to account for temporal trends adds substantial rigor to this study, since the majority of these patients were cared for by house staff whose experience and efficiency vary throughout the academic year. Furthermore, subsequent multivariate analysis demonstrated consistent findings after adjustment for comorbid illness and

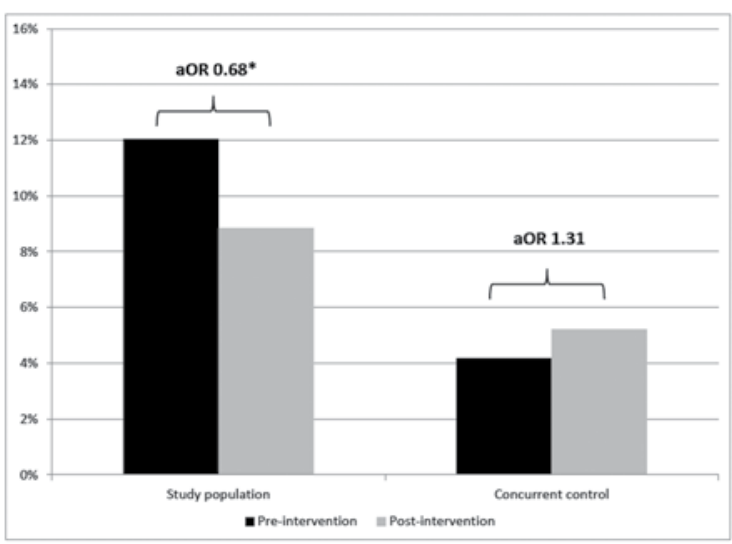

FIG. 2.: Inhospital mortality

aAdjusted odd ratio for intervention group was significant at $P<0.05$. Model includes adjustment for age, gender, payer, admitting team, ICU status, time of admission, and modified Elixhauser index.

NOTE: Inhospital mortality in study population pre- and postintervention compared to concurrent control group of transfer patients routed through cardiac transfer center during same time period who did not receive the intervention. Abbreviation: ICU, intensive care unit. 
several other hospital and patient-level confounders.

This study has several limitations. The primary limitation is its nonrandomized design. Patient characteristics were stable across multiple variables before and after implementation, but it is possible that another confounding factor was responsible for observed improvements. Likewise, we collected data for only 6 rather than 12 months during the postintervention time period, which limited our sample size and statistical power. This was chosen because a significant restructuring of resident duty hours occurred in spring 2011 that had the potential to affect all measures studied. ${ }^{20,21} \mathrm{Fi}$ nally, we did not collect data on accuracy of the information provided in the handover tool or end-user utilization and were unable to account for effects of these.

Since implementation in 2010, this process for interhospital transfers at VUH remains the same, although the volume of incoming transfers has significantly increased. Electronic handover tools with similar structure and content have since been adopted for patients being transferred to the emergency department or directly admitted from clinic. As VUH moves in the coming years from a locally developed

\section{References}

1. Coleman EA, Min SJ, Chomiak A, Kramer AM. Posthospital care transitions: pat terns, complications, and risk identification. Health Serv Res. 2004;39:1449-1465.

2. Iwashyna TJ, Christie JD, Moody J, Kahn JM, Asch DA. The structure of critical care transfer networks. Med Care. 2009;47:787-793.

3. Durairaj L, Will JG, Torner JC, Doebbeling BN. Prognostic factors for mortality following interhospital transfers to the medical intensive care unit of a tertiary referral center. Crit Care Med. 2003;31:1981-1986.

4. Golestanian E, Scruggs JE, Gangnon RE, Mak RP, Wood KE. Effect of interhospital transfer on resource utilization and outcomes at a tertiary care referral center. Crit Care Med. 2007;35:1470-1476.

5. Flabouris A, Hart GK, George C. Outcomes of patients admitted to tertiary intensive care units after interhospital transfer: comparison with patients admitted from emergency departments. Crit Care Resusc. 2008;10:97-105.

6. Combes A, Luyt CE, Trouillet JL, Chastre J, Gibert C. Adverse effect on a referral intensive care unit's performance of accepting patients transferred from another intensive care unit. Crit Care Med. 2005;33:705-710.

7. Rosenberg AL, Hofer TP, Strachan C, Watts CM, Hayward RA. Accepting critically ill transfer patients: adverse effect on a referral center's outcome and benchmark measures. A Intern Med. 2003;138:882-890.

8. Horwitz LI, Moin T, Krumholz HM, Wang L, Bradley EH. Consequences of inadequate sign-out for patient care. Arch Intern Med. 2008;168:1755-1760.

9. Starmer AJ, Sectish TC, Simon DW, et al. Rates of medical errors and preventable adverse events among hospitalized children following implementation of a resident handoff bundle. JAMA. 2013;310:2262-2270.

10. Arora VM, Manjarrez E, Dressler DD, Basaviah P, Halasyamani L, Kripalani S. Hospitalist handoffs: a systematic review and task force recommendations. J Hosp Med. 2009;4:433-440.

11. Bosk EA, Veinot T, Iwashyna TJ. Which patients and where: a qualitative study of patient transfers from community hospitals. Med Care. 2011;49:592-598. electronic medical record to a national vendor, there will be an opportunity to transform this tool into an electronic template that will easily share data between institutions and further enhance communication.

Interhospital transfer patients represent a high-risk population whose unique handover needs have not been adequately measured or addressed. Our investigation demonstrated that a standardized handover aid can be implemented across a broad transfer network and may contribute to reductions in LOS and mortality. Further study is warranted to confirm these findings and assess the effect on other clinical outcomes.

Disclosures: This material is based upon work supported by the Office of Academic Affiliations, Department of Veterans Affairs, VA National Quality Scholars Program, and was made possible by the use of the facilities at VA Tennessee Valley Healthcare System, Nashville, Tennessee. The views expressed in this article are those of the authors and do not necessarily reflect the position or policy of the Department of Veterans Affairs or the US government. Additionally, this publication was supported in part by CTSA award No. UL1TR000445 from the National Center for Advancing Translational Sciences. Its contents are solely the responsibility of the authors and do not necessarily represent official views of the National Center for Advancing Translational Sciences or the National Institutes of Health.

12. Herrigel DJ, Carroll M, Fanning C, Steinberg MB, Parikh A, Usher M. Interhospital transfer handoff practices among US tertiary care centers: a descriptive survey. J Hosp Med. 2016;11:413-417.

13. Kripalani S, LeFevre F, Phillips CO, Williams MV, Basaviah P, Baker DW. Deficits in communication and information transfer between hospital-based and primary care physicians. JAMA. 2007;297:831-841.

14. Gandara E, Moniz TT, Ungar J, et al. Deficits in discharge documentation in patients transferred to rehabilitation facilities on anticoagulation: results of a systemwide evaluation. Jt Comm J Qual Patient Saf. 2008;34:460-463.

15. Haig KM, Sutton S, Whittington J. SBAR: a shared mental model for improving communication between clinicians. Jt Comm J Qual Patient Saf. 2006;32:167-175.

16. van Walraven C, Austin PC, Jennings A, Quan H, Forster AJ. A modification of the Elixhauser comorbidity measures into a point system for hospital death using administrative data. Med Care. 2009;47:626-633.

17. Wagner AK, Soumerai SB, Zhang F, Ross-Degnan D. Segmented regression analysis of interrupted time series studies in medication use research.J Clin Pharm Ther. 2002;27:299-309.

18. Malpass HC, Enfield KB, Keim-Malpass J, Verghese GM. The interhospital medical intensive care unit transfer instrument facilitates early implementation of critical therapies and is associated with fewer emergent procedures upon arrival. $J$ Intensive Care Med. 2015;30:351-357.

19. National Academy of Sciences. Institute of Medicine. Crossing the Quality Chasm: A New Health System for the 21st Century. March 2005:1-360. Washington, DC.

20. Theobald CN, Stover DG, Choma NN, et al. The effect of reducing maximum shift lengths to 16 hours on internal medicine interns' educational opportunities. Acad Med. 2013;88:512-518.

21. Choma NN, Vasilevskis EE, Sponsler KC, Hathaway J, Kripalani S. Effect of the ACGME 16-hour rule on efficiency and quality of care: duty hours 2.0. JAMA Intern Med. 2013;173:819-821. 